\section{A Fast Method for Steady-State Memristor Crossbar Array Circuit Simulation}

Rui Xie ${ }^{1}$, Mingyang Song ${ }^{1}$, Junzhuo Zhou ${ }^{1}$, Jie Mei ${ }^{1}$, Quan Chen*1 (Corresponding Author)

${ }^{1}$ School of Microelectronics, Southern University of Science and Technology

\begin{abstract}
In this work we propose an effective preconditioning technique to accelerate the steady-state simulation of large-scale memristor crossbar arrays (MCAs). We exploit the structural regularity of MCAs to develop a specially-crafted preconditioner that can be efficiently evaluated utilizing tensor products and block matrix inversion. Numerical experiments demonstrate the efficacy of the proposed technique compared to mainstream preconditioners.
\end{abstract}

Index Terms-Memristor, Neural Network, Crossbar Circuits, Preconditioner, GMRES

\section{INTRODUCTION}

MCAs (Memristor Crossbar Arrays) [1] has gained substantial attention recent years because of its potential application in high-performance AI hardware and neuromorphic computing [2], calling for efficient circuit simulation tools. However, efficient simulation of MCAs has become increasingly challenging. The expected size of MCA is growing rapidly to accommodate the millions of weights involved in state-of-the-art neural networks [3]. Furthermore, a large amount of simulations are needed for statistical characterization or if the training/inference procedures are to be studied at circuit simulation level.

Existing steady-state simulation of MCA circuits is often done by SPICE, in which a sparse linear system resulted from the modified nodal analysis (MNA) must be solved in each Newton iteration. The matrix size can be huge, e.g., a $1024 \times 1024$ MCA leads to a matrix size $>10^{6}$, resulting in severe bottlenecks in time and memory consumption if direct solvers are used. Iterative solvers can improve the scalability, but existing general-purpose - preconditioners [4] are often not adequately efficient for largescale MCA circuits. In this work, we leverage the special topology of MCAs to develop an efficient preconditioning technique to accelerate the steady-state simulation of MCAs. Specifically, the preconditioner has the following features:

1) It takes advantages of the topological regularity of MCAs to generate special block structures;

2) Its inverse and application to vectors can be efficiently evaluated by Kronecker product and block matrix inversion formula.

\section{BACKGROUND}

A voltage-controlled MCA is illustrated by Fig 3. It can be divided into three parts: the top metal layer, the middle vertical memristor devices and the bottom metal layer, as shown in Fig. 1(a), Fig. 1(b) and Fig. 1(c). The top and the bottom metal layers are assumed to be two uniform grids, with equal conductance for each grid segment (but the conductance per segment can be different for the two layers). The memristor devices lie between the corresponding points of the two grids.

The steady-state MNA equation is given in (1), where $G_{t}$ and $G_{b}$ are the conductance matrices for the top and the bottom layers.
$V_{t}$ and $V_{b}$ are the corresponding nodal voltage unknowns. $I_{t}$ and $I_{b}$ are the nonlinear functions of $V_{t}$ and $V_{b}$ relating the steadystate memristor currents to the applied voltages. Additionally, $Y_{t}$ and $Y_{b}$ are the boundary conditions. All of them combine to form the matrix equation (9). The whole nonlinear equation is solved by the Newton's method (3) with the Jacobian matrix given in (4).
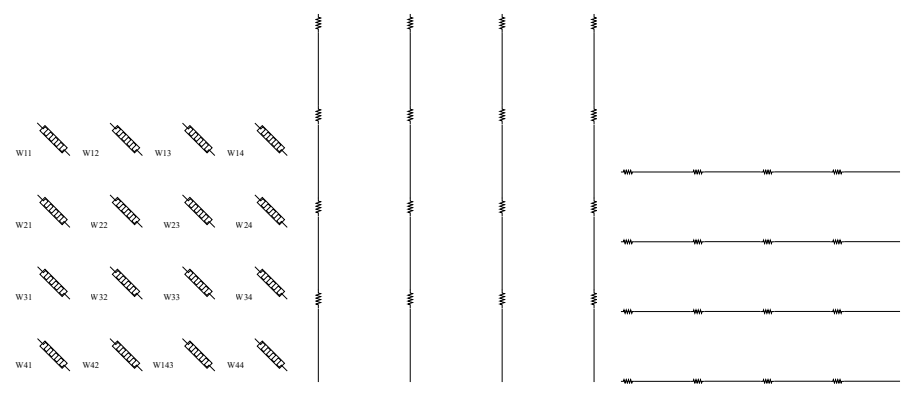

(a) The middle layer of (b) The top metal layer (c) The bottom metal memristors. with conductance repre- layer with conductance
sented as $G_{t}$.

Fig. 1: A division of MCA crossbar

$$
\begin{aligned}
& G_{t} * V_{t}+I_{t}\left(V_{t}, V_{b}\right)-Y_{t}=0 \\
& G_{b} * V_{b}-I_{b}\left(V_{t}, V_{b}\right)-Y_{b}=0 \\
& G_{t}=I \otimes G \\
& G_{b}=G \otimes I \\
& J\left(\vec{V}_{n}\right) *\left(\vec{V}_{n+1}-\vec{V}_{n}\right)=\left(-F\left(\vec{V}_{n}\right)\right) \\
& J=F^{\prime}=\left[\begin{array}{cc}
G_{t} & 0 \\
0 & G_{b}
\end{array}\right]+\left[\begin{array}{cc}
\frac{\partial I_{t}}{\partial V_{t}} & \frac{\partial I_{t}}{\partial V_{b}} \\
\frac{-\partial I_{b}}{\partial V_{t}} & \frac{-\partial I_{b}}{\partial V_{b}}
\end{array}\right]
\end{aligned}
$$

\section{The Proposed Preconditioning TechniQue}

In this work we focus on using the iterative solution method of GMRES (Generalized minimal residual method) to solve the sparse total Jacobian matrix in (4). $J$ consists of two parts: the linear conductance matrix from the interconnect and the nonlinear Jacobian from the I-V functions of the memristor devices.

\section{A. Preconditioner Formulation}

Firstly, we choose a particular indexing scheme to give $J$ a special sparsity structure. The top and the bottom layers both use natural indexing, but the directions are perpendicular to each other, as illustrated in Fig. 1] There are two reasons for this choice: 1) the four blocks in the nonlinear Jacobian matrix are now all diagonal; 2) by assuming equal conductances for all segments at the same layer, we can rewrite the top and the bottom linear conductance matrices $G_{t}, G_{b} \in \mathcal{R}^{n^{2} \times n^{2}}$ into Kronecker products (2a) and (2b), where $G \in \mathcal{R}^{n \times n}$ is the conductance matrix of single row or column (8).

$$
P=\left[\begin{array}{cc}
G_{t}+a_{1} I & -a_{1} I \\
-a_{2} I & G_{b}+a_{2} I
\end{array}\right]
$$


Next, we develop a special preconditioner of the form in (5) with the same block structure. The $a_{1}$ and $a_{2}$ are the mean of the diagonal elements of $\frac{\partial I_{t}}{\partial V_{b}}$ and $\frac{-\partial I_{b}}{\partial V_{b}}$, which can be considered as the average conductance of the memristor devices. Notice that $\frac{\partial I_{t}}{\partial V_{t}}$ and $\frac{\partial I_{t}}{\partial V_{b}}$ are opposite, as well as $\frac{-\partial I_{b}}{\partial V_{t}}$ and $\frac{-\partial I_{b}}{\partial V_{b}}$, since $V_{b}$ and $V_{t}$ are the voltages across the memristors.

\section{B. Fast Evaluation of Preconditioner}

$$
\begin{aligned}
& {\left[\begin{array}{cc}
A & B \\
C & D
\end{array}\right]^{-1}=\left[\begin{array}{cc}
-M^{-1} D B^{-1} & M^{-1} \\
B^{-1}+B^{-1} A M^{-1} D B^{-1} & -B^{-1} A M^{-1}
\end{array}\right] } \\
& M=\left(C-D B^{-1} A\right) \\
&=\left(-a_{2} I-\left(G_{b}+a_{2} I\right)\left(-a_{1} I\right)^{-1}\left(G_{t}+a_{1} I\right)\right) \\
&=\left(-a_{2} I+\frac{1}{a_{1}}\left(G \otimes I+a_{2} I\right)\left(I \otimes G+a_{1} I\right)\right) \\
&=\left(-a_{2} I+\frac{1}{a_{1}}\left(G_{2} \otimes I\right)\left(I \otimes G_{1}\right)\right) \\
&=\left(-a_{2} I+\frac{1}{a_{1}}\left(G_{2} \otimes G_{1}\right)\right)
\end{aligned}
$$

It is important to have a fast scheme to evaluate $P^{-1} v$. We first apply the Woodbury block matrix inversion identity (6). Note that the off-diagonal blocks $B$ and $C$ are just identity matrices whose inverse is trivial. The core operation is to obtain $M^{-1} v=$ $\left(C-D B^{-1} A\right)^{-1} v$.

To this end, we rewrite $M$ into (7), with $G_{1}$ and $G_{2}$ given in (10a) and 10b. In typical MCAs, the memristor conductance is generally much smaller than that of interconnects. Therefore, one can drop the first term on the right hand side of (11) and approximate $M$ as in (15) and (14). To compute (16), where $\hat{g}_{i, j}^{2}$ is the element of $\widehat{G_{2}}$. Vector $v$ can be rearranged by $(17), V_{j}$ represent the $j^{\text {th }}$ column of $\hat{V}$. Consider the $j^{\text {th }}$ row in (18). Finally, we can deduce original equation to (19).

\section{NumericAl RESUlTS}

In the following tests, the top and bottom wire conductance $g$ per segment are normalized to 1 . We adopt the Yakopcic model [5] as the RRAM model. Since the proposed method is expected to handle RRAM devices of various states, we obtain the conductance matrix of RRAM by randomly setting the internal state variable of their model, with a maximum conductance being 0.4 to meet the approximation condition (12). The GMRES solver from Scipy is used with a uniform relative tolerance of $10^{-6}$.

Fig. 2 shows the residual history of GMRES with and without the proposed preconditioner. The test case is a $128 \times 128$ crossbar with the matrix dimension of $32768 \times 32768$. It can be seen that the proposed preconditioner drastically accelerates the convergence of GMRES.

Fig. 4 compares the iteration number for MCAs of five difference sizes $(32 \times 32,64 \times 64,128 \times 128,256 \times 256$ and $512 \times 512)$. The matrix sizes are labeled on the lines and the corresponding iteration numbers summarized in the table. It is clear that the computational saving from the proposed preconditioner grows rapidly as the matrix size increases.

Table II compares the proposed preconditioner against other mainstream preconditioners such as the Jacobi and the ILU pre-

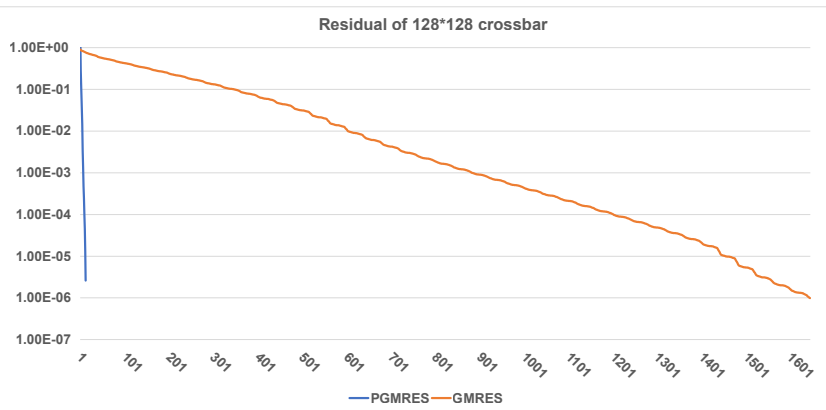

Fig. 2: Residual of preconditioned GMRES (PGMRES) and baseline GMRES for $128 \times 128$ crossbar .

TABLE I. Comparison of Total CPU Time Consumption and Iteration Steps to Coverage

\begin{tabular}{ccccccccc}
\hline & \multicolumn{2}{c}{$\begin{array}{c}\text { Before } \\
\text { Preconditioned }\end{array}$} & \multicolumn{2}{c}{$\begin{array}{c}\text { Jacobi } \\
\text { Preconditioner }\end{array}$} & \multicolumn{2}{c}{$\begin{array}{c}\text { ILU } \\
\text { Preconditioner }\end{array}$} & \multicolumn{2}{c}{$\begin{array}{c}\text { Our } \\
\text { Preconditioner }\end{array}$} \\
\hline $\begin{array}{c}\text { Crossbar } \\
\text { Dimension (n) }\end{array}$ & Steps & $\begin{array}{c}\text { CPU time } \\
\text { consumption/s }\end{array}$ & Steps & $\begin{array}{c}\text { CPU time } \\
\text { consumption/s }\end{array}$ & Steps & $\begin{array}{c}\text { CPU time } \\
\text { consumption/s }\end{array}$ & Steps & $\begin{array}{c}\text { CPU time } \\
\text { consumption/s }\end{array}$ \\
\hline $16 * 16$ & 67 & 0.01596 & 60 & 0.00897 & 3 & 0.00598 & 11 & 0.00299 \\
\hline $32 * 32$ & 227 & 0.05785 & 203 & 0.03092 & 15 & 0.01396 & 11 & 0.02194 \\
\hline $64 * 64$ & 678 & 0.30377 & 551 & 0.14319 & 111 & 0.14561 & 13 & 0.09275 \\
\hline $128 * 128$ & 1645 & 1.97858 & 1610 & 0.95511 & 308 & 1.24064 & 13 & 0.57907 \\
\hline $256 * 256$ & 7526 & 28.51413 & 5361 & 23.41768 & 589 & 11.05555 & 13 & 4.77912 \\
\hline $512 * 512$ & 22801 & 331.31852 & 19273 & 324.20583 & 3120 & 309.91122 & 17 & 225.12859 \\
\hline
\end{tabular}

conditioner. The iteration number and the total CPU runtime are recorded for MCAs of different sizes. For small cases, the three types of preconditioners perform comparably well. For larger cases, the proposed preconditioner requires much fewer iterations than the other two preconditioners. The runtime reduction is less significant due to the evaluation of preconditioner not being fully optimized. Future efforts will be devoted to speed up this part.

\section{CONClusion}

We have devised an efficient preconditioner for fast iterative solution of the Jacobian matrices appearing in steady-state MCA simulation. The preconditioner leverages the special sparsity pattern in the Jacobian matrices resulted from a deliberately crafted indexing scheme. Tensor product and block matrix inversion techniques are utilized to significantly accelerate the preconditioner evaluations during the iterative solutions. Numerical results have demonstrated the efficacy of the proposed preconditioner.

\section{REFERENCES}

[1] Chua, L. (1971). Memristor-the missing circuit element. IEEE Transactions on circuit theory, 18(5), 507-519.

[2] Zhang, F., \& Hu, M. (2020, November). CCCS: customized spice-level crossbar-array circuit simulator for in-memory computing. In Proceedings of the 39th International Conference on Computer-Aided Design (pp. 1-8). [2] A. N. Expert, A Book He Wrote, His Publisher, 1989.

[3] Truong, S. N., Van Pham, K., Yang, W., \& Min, K. S. (2016, October). Memristor circuits and systems for future computing and bio-inspired information processing. In 2016 IEEE Biomedical Circuits and Systems Conference (BioCAS) (pp. 456-459). IEEE.

[4] Ferronato, M.. Preconditioning for sparse linear systems at the dawn of the 21st century: History, current developments, and future perspectives. International Scholarly Research Notices, 2012.

[5] C. Yakopcic, T. M. Taha, G. Subramanyam, R. E. Pino and S. Rogers, "A Memristor Device Model," in IEEE Electron Device Letters, vol. 32, no. 10, pp. 1436-1438, Oct. 2011, 


\section{A. Equations and Figures}

$$
\begin{aligned}
& G=\left[\begin{array}{ccccc}
2 g & -g & \ldots & 0 \\
-g & 2 g & \cdots & \\
\vdots & \ddots & \vdots \\
0 & \cdots & 2 g & -g \\
& & & -g & 2 g
\end{array}\right] \\
& F=\left[\begin{array}{cc}
G_{t} & 0 \\
0 & G_{b}
\end{array}\right] *\left[\begin{array}{c}
V_{t} \\
V_{b}
\end{array}\right]+\left[\begin{array}{c}
I_{t} \\
-I_{b}
\end{array}\right]-\left[\begin{array}{c}
Y_{t} \\
Y_{b}
\end{array}\right]=0
\end{aligned}
$$

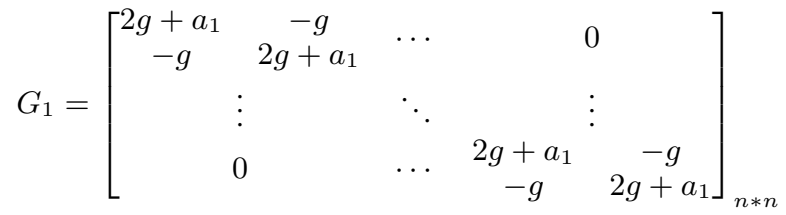

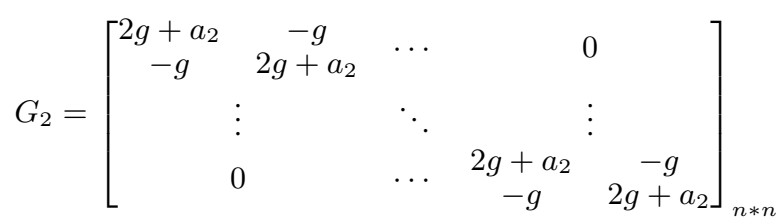

$$
\begin{aligned}
& \operatorname{diag}(M)=\left(-a_{2}+\frac{1}{a_{1}}\left(2 g+a_{2}\right)\left(2 g+a_{1}\right)\right) \\
& 20 a_{2}<\frac{1}{a_{1}}\left(2 g+a_{2}\right)\left(2 g+a_{1}\right) \\
& M \cong \hat{M}=\widehat{G_{2}} \otimes G_{1} \\
& \begin{array}{c}
\widehat{G_{2}}=\left[\begin{array}{ccccc}
\frac{2 g+a_{2}}{a_{1}} & -g & & & \\
-g & \frac{2 g+a_{2}}{a_{1}} & \cdots & 0 & \\
\vdots & & \ddots & \vdots \\
0 & & \cdots & \frac{2 g+a_{2}}{a_{1}} & \frac{2 g}{a_{1}}
\end{array}\right]_{n * n} \\
M^{-1} \cong \hat{M}^{-1}={\widehat{G_{2}}}^{-1} \otimes G_{1}^{-1}
\end{array} \\
& \left({\widehat{G_{2}}}^{-1} \otimes G_{1}^{-1}\right) v \\
& =\left({\widehat{G_{2}}}^{-1} \otimes G_{1}^{-1}\right)\left[\begin{array}{c}
v_{1,1} \\
v_{1,2} \\
\vdots \\
v_{1, n-1} \\
v_{1, n} \\
v_{2,1} \\
\vdots \\
v_{2, n} \\
\vdots \\
v_{n, n}
\end{array}\right] \\
& =\left[\begin{array}{ccc}
\frac{1}{\hat{g}_{1,1}^{2}} * G_{1}^{-1} & \cdots & \frac{1}{\hat{g}_{1, n}^{2}} * G_{1}^{-1} \\
\vdots & \ddots & \vdots \\
\frac{1}{\hat{g}_{n, 1}^{2}} * G_{1}^{-1} & \cdots & \frac{1}{\hat{g}_{n, n}^{2}} * G_{1}^{-1}
\end{array}\right]\left[\begin{array}{c}
v_{1,1} \\
v_{1,2} \\
\vdots \\
v_{1, n-1} \\
v_{1, n} \\
v_{2,1} \\
\vdots \\
v_{2, n} \\
\vdots \\
v_{n, n}
\end{array}\right]
\end{aligned}
$$

Fig. 4: Iteration number to coverage of dimension of crossbar $32 \times 32,64 \times 64,128 \times 128,256 \times 256$ and $512 \times 512$.

Fig. 3: A general MCA is shown with BL (bit line) and WL (word line).

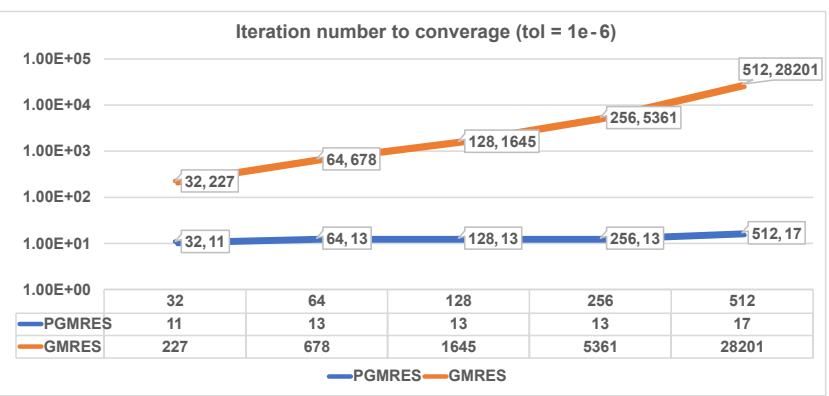

$32 \times 32,64 \times 64,128 \times 128,256 \times 256$ and $512 \times 512$. 